\title{
ADHERENCIA A LA DIETA MEDITERRÁNEA EN ESTUDIANTES UNIVERSITARIOS DEL NORTE DE MÉXICO.
}

\author{
ADHERENCE TO THE MEDITERRANEAN DIET IN UNIVERSITY STUDENTS OF SCIENCES OF PHYSICAL \\ CULTURE.
}

Delgado Flores Monserrat*, Martínez Martínez Cesar Augusto*, Klassen Merancio Max Eduardo*, Guedea Delgado Julio Cesar*, Muñoz-Daw María de Jesús*.

*Universidad Autónoma de Chihuahua, Facultad de Ciencias de la Cultura Física. México.

Citation: Delgado Flores M., Martínez Martínez C.A., Klassen Merancio M.E., Guedea Delgado J.C., Muñoz-Daw M.J. (2021) Adherencia a la dieta mediterránea en estudiantes universitarios del norte de México. Revista Salud Pública y Nutrición, 20 (2), 22-31.

Editor: Esteban G. Ramos Peña, Dr. CS., Universidad Autónoma de Nuevo León, Facultad de Salud Pública y Nutrición, Monterrey Nuevo León, México. Copyright: (C2021 Delgado Flores M., et al. This is an open-access article distributed under the terms of Creative Commons Attribution License [CC BY 4.0], which permits unrestricted use, distribution, and reproduction in any medium, provided the original author and source are credited.

Competing interests: The authors have declared that no competing interests exist.

DOI: $\underline{\text { https://doi.org/10.29105/respyn20.2-3 }}$

Recibido: 02 de septiembre 2020; Aceptado: 08 de marzo 2021

Email: cesar mtz15@hotmail.com 


\title{
ADHERENCIA A LA DIETA MEDITERRÁNEA EN ESTUDIANTES UNIVERSITARIOS DEL NORTE DE MÉXICO.
}

\author{
Delgado Flores Monserrat*, Martínez Martínez Cesar Augusto*, Klassen Merancio Max Eduardo*, Guedea \\ Delgado Julio Cesar*, Muñoz-Daw María de Jesús*.
}

* Universidad Autónoma de Chihuahua, Facultad de Ciencias de la Cultura Física. México.

\section{RESUMEN}

Introducción. La dieta mediterránea es un patrón alimentario altamente reconocido como beneficioso para la salud. Objetivo: Evaluar y comparar por sexo la adherencia a la dieta mediterránea de los estudiantes universitarios de la Facultad de Ciencias de la Cultura Física de la Universidad Autónoma de Chihuahua. Material y Método: Estudio comparativo, descriptivo y transversal en el que participaron 165 estudiantes, 88 mujeres y 77 hombres, entre 22 y 34 años. Se analizó el peso, estatura, índice de masa corporal y la adherencia a la dieta mediterránea utilizando el cuestionario KIDMED según el sexo. Se utilizaron las pruebas estadísticas de t de Student, U-Mann Whitney y Chi cuadrado. Resultados: Se clasificaron con sobrepeso el $30.7 \%$ de las mujeres y el $46.8 \%$ de los hombres con diferencia estadística $(p<0.04)$ y con obesidad $9.1 \%$ en ambos sexos. En la adherencia a la dieta mediterránea el $33.9 \%$ clasificó con muy baja, $48.5 \%$ con necesidad de ajustes a los patrones mediterráneos y $17.6 \%$ como óptima, no se reflejó diferencias por sexo $(p<0.788)$. Conclusiones: La adherencia a la dieta mediterránea requiere de ajustes, requiriendo hacer mayor promoción de los beneficios a corto y largo plazo que ofrece la dieta mediterránea respecto a la dieta occidental.

Palabras Clave: Alimentación, nutrición, dieta mediterránea, universitarios.

\section{ABSTRACT}

Introduction: The Mediterranean diet is a feeding pattern highly recognized as beneficial for health. Objective: To evaluate and compare by gender the adherence to the Mediterranean diet of university students at the Faculty of Science of the Physical Culture of Universidad Autónoma de Chihuahua. Material and method: It was a comparative, descriptive and crosssectional study. 165 students participated, 88 women and 77 men, between 22 and 34 years old. The variables of weight, height, body mass index and the adherence to the Mediterranean diet by gender were analyzed. The statistical tests of Student's t-test, Mann-Whitney-U and Chi-squared were used. Results: $30.7 \%$ of the women and $46.8 \%$ of the men were classified as overweight with statistical difference $(p<0.04)$ and $9.1 \%$ obesity in both sexes. In adherence to the Mediterranean diet, the $33.9 \%$ classified as very low, $48.5 \%$ as needing adjustments to the Mediterranean patterns and $17.6 \%$ as optimal, no differences by gender were reflected $(p<0.788)$. Conclusions: Adherence to the Mediterranean diet requires adjustments, requiring greater promotion of the short and long-term benefits offered by the Mediterranean diet compared to the Western diet.

Key words: Feeding, nutrition, mediterranean diet, university students. 


\section{Introducción}

La dieta mediterránea fue declarada Patrimonio Inmaterial de la Humanidad por la Organización de las Naciones Unidas para la Educación, la Ciencia y la Cultura (UNESCO) en 2013, reconocida como una filosofía de vida, fruto de las relaciones interculturales que comparte conocimientos, prácticas, tradiciones de los cultivos y cosechas agrícolas, la pesca y la cría de animales, la forma de conservar, cocinar, compartir y consumir los alimentos, implicando un intercambio social. La dieta mediterránea nutricionalmente es reconocida y recomendada por ser alta en antioxidantes, en fibra, en aceites omega 3, compuestos fitoactivos como el licopeno, lupeol, quercetina, genisteína, carnosol y resveratrol, aportes adecuados de minerales, vitaminas, proteínas y energía (Escaffi et al., 2016).

La dieta mediterránea se caracteriza por el consumo de platillos con alto contenido de verduras y frutas, consumo frecuente de frutos secos, cereales integrales y bajo consumo de alimentos de origen animal, en los que se prefiere el pescado, las aves de corral y lácteos bajos en grasa (Díaz et al., 2007). Es por este motivo que la dieta mediterránea es reconocida a nivel internacional por ser una dieta saludable, se considera como un tratamiento preventivo y primario para enfermedades relacionadas con la inflamación crónica de acuerdo con Dussaillant et al. (2016) y que puede ser utilizada para disminuir o controlar enfermedades como el síndrome metabólico, cardiovasculares, diabetes, hipertensión y mejorar la calidad de vida (Babio et al., 2009; Serra \& Ortiz, 2018).

En contraste, la dieta occidental conformada por dos acontecimientos históricos, la revolución agrícola y la Industrial, se caracteriza por comidas rápidas, altas en carnes rojas, harinas refinadas, lácteos y azúcares refinados, lo que ha traído un aumento en los problemas de salud relacionados con la dislipidemia que ocasionan enfermedades cardiovasculares, diabetes e hipertensión. Según Peñalvo et al. (2015), ésta alimentación también se encuentra asociada con el 35\% de las muertes por cáncer y específicamente con el 10 y $12 \%$ del cáncer de próstata (Ferrís et al., 2012).

En el estudio de Arriaga \& Cruz (2019) compararon 3 dietas: baja en grasa, dieta mediterránea y baja en carbohidratos, la dieta mediterránea y la dieta baja en carbohidratos demostraron ser una alternativa efectiva para la reducción del peso corporal en comparación con la dieta baja en grasa.

Durante el periodo universitario los jóvenes se encuentran en un momento crítico, ya que el desarrollo de sus estilos de vida impactará en su desempeño y salud a futuro (Gómez et al., 2016). Convirtiéndose en un grupo vulnerable desde el punto de vista nutricional (Landeros et al., 2018), ya que en la etapa universitaria existen muchos cambios como la exigencia de los estudios, cambios psicosociales y aumento de estancia universitaria. Esta etapa se caracteriza además por la transición de la adolescencia a la edad adulta, lo que conlleva aumento de la independencia, autonomía y responsabilidad de los universitarios, los cuales toman decisiones sobre el “¿cómo?, ¿qué?, ¿dónde? y ¿cuándo comer?", como lo comentan Van Kim et al. (2012), todo esto a veces sin tener conocimiento de su salud y necesidades alimentarias, este desconocimiento los lleva a malos hábitos nutricios como ayunos, saltearse comidas, comer a horas inadecuadas, preferencia por las comidas rápidas, elevado consumo de alcohol (Arroyo et al., 2006) y alimentos de origen animal, por lo que el perfil calórico de los mismos se aleja de lo recomendado (Ponce et al., 2011).

La alimentación es uno de los factores asociados a la actividad física y el deporte para mantener la salud y el rendimiento físico adecuado, proporcionando los micronutrientes requeridos para que el atleta en un estado saludable potencialice sus funciones metabólicas, así maximizará sus resultados en el entrenamiento. Una baja disponibilidad de la cantidad de energía en la dieta puede resultar en una perdida no deseada de masa muscular, disfunción hormonal, baja densidad ósea, aumento de fatiga, lesiones con una recuperación prolongada y otras enfermedades (Thomas et al., 2016).

El cuestionario KIDMED es una herramienta para evaluar la adherencia a la dieta mediterránea en niños y jóvenes, que fue desarrollado y validado por Serra et al. (2004). La prueba se basa en los principios del patrón dietético mediterráneo, a través de la evaluación del consumo de 16 componentes, ayudando a identificar personas con cantidades adecuadas de la ingesta de nutrientes (índice más 
alto) y con hábitos poco saludables (índice más bajo) (García et al., 2015).

El objetivo de esta investigación fue evaluar la adherencia de la dieta mediterránea en los estudiantes de séptimo y octavo semestre de la Facultad de Ciencias de la Cultura Física de la Universidad Autónoma de Chihuahua a través del cuestionario KIDMED para poder efectuar la comparación entre hombres y mujeres participantes.

\section{Material y Método}

El diseño utilizado fue cuantitativo, descriptivo y transversal. La muestra fue no aleatoria, se realizó un muestro por conveniencia y estuvo conformada por 165 estudiantes universitarios de $7^{\circ}$ y $8^{\circ}$ semestre de la Facultad de Ciencias de la Cultura Física de los cuales $88(53 \%)$ son mujeres y $77(47 \%)$ hombres, se excluyeron únicamente los estudiantes que no asistieron el día de la evaluación; por lo que la muestra corresponde al $87 \%$ de los estudiantes de $7^{\circ}$ y $8^{\circ}$ la facultad. Para la realización de este estudio se solicitó permiso a las autoridades universitarias. A los estudiantes se les dio una amplia explicación sobre la investigación y a los que aceptaron participar voluntariamente se les repartió el cuestionario KIDMED.

La edad y el sexo se recogió en el mismo cuestionario KIDMED. La muestra se caracterizó con antropometría básica de masa corporal y estatura, las cuales se realizaron en el laboratorio de antropometría y con la técnica de la Sociedad Internacional para el Avance de la Cineantropometría (ISAK), se midió la masa corporal con el mínimo de ropa, en la báscula digital Tanita modelo BF-680W (TANITA Corporation Inc., Airlington Heights, Illinois, Estados Unidos) la estatura máxima en posición de bipedestación sin calzado y con el peso distribuido equitativamente y la cabeza en posición Frankfort, se midió con el estadímetro marca Seca 206 (SECA Ltd., Hamburgo, Alemania) fijo en una pared lisa.

El índice de masa corporal (IMC) se calculó dividiendo la masa corporal en kilogramos entre la estatura en metros al cuadrado $(\mathrm{kg} / \mathrm{m} 2)$, el cual permitió clasificar a los sujetos de estudio en bajo peso cuando el IMC es menor a 18.5 , normopeso cuando se encuentra entre 18.5 y 24.9 , sobrepeso de 25 a 29.9 y obesidad de 30 o mayor WHO (1995).
Para evaluar la adherencia a la dieta mediterránea se utilizó el cuestionario de KIDMED de Serra et al., (2004), que consta de 16 preguntas, a las cuales se les asigna una puntuación, las preguntas que presentan adherencia a la DIETA MEDITERRÁNEA suman un punto, las preguntas con connotación negativa al patrón de la misma restan un punto y el resultado se clasifica en tres niveles: calidad de dieta óptima, con una puntuación mayor o igual a ocho, necesidad de ajustar la ingesta a los patrones mediterráneos, de cuatro a siete puntos y muy baja calidad en la dieta, menor o igual a tres puntos, el cual fue auto-aplicado en un tiempo aproximado de 10 minutos.

Las variables de estudio fueron la adherencia a la dieta mediterránea, peso, talla, índice de masa corporal y la variable categórica fue el sexo.

Para el análisis estadístico se utilizó el paquete estadístico del SPSS v21, para las variables cuantitativas de antropometría se determinó la normalidad con la prueba de Kolmogorov-Smirnov, para la estadística descriptiva y para comparar medias y medianas se utilizó la prueba de $\mathrm{t}$ de Student para los datos normales y U-Mann Whitney para los datos no normales, así mismo, se determinaron frecuencias y porcentajes, para evaluar diferencias de datos no paramétricos, en la clasificación del IMC y en la adherencia a la dieta mediterránea por sexo se utilizó la prueba de Chi cuadrado al $95 \%$ de confianza al igual que las diferencias estadísticas de preguntas del cuestionario. Este estudio se realizó bajo los principios éticos de la declaración de Helsinki.

\section{Resultados}

De los estudiantes universitarios que participaron el $53.3 \%$ fueron mujeres y el $46.7 \%$ hombres, con edades comprendidas entre 22 y 34 años, con una mediana de 23 años. Los valores obtenidos para las medidas antropométricas como la masa corporal y la estatura tuvieron diferencias estadísticas propias del sexo, mayores en los hombres $(p<0.001)$, en el IMC el promedio de las mujeres corresponde a normopeso y en los hombres a sobrepeso (tabla 1). 
Tabla 1. Datos antropométricos de los estudiantes universitarios según el sexo

\begin{tabular}{lcccc}
\hline & \multicolumn{2}{c}{ Mujeres $(\mathrm{n}=88)$} & \multicolumn{2}{c}{ Hombres $(\mathrm{n}=77)$} \\
& media & $\mathrm{DE}$ & Media & $\mathrm{DE}$ \\
\hline $\mathrm{IMC}\left(\mathrm{kg} / \mathrm{m}^{2}\right)^{*}$ & 24 & 4.6 & 25.6 & 3.6 \\
Estatura $(\mathrm{m})^{*}$ & 1.63 & 1.5 & 1.74 & 1.5 \\
& & & & \\
& Mediana & RIQ & Mediana & RIQ \\
\cline { 2 - 5 } Masa Corporal $(\mathrm{kg})^{*}$ & 64.6 & 13.8 & 77.8 & 13.1 \\
\hline
\end{tabular}

Fuente: Encuesta.

*Estadística descriptiva de los estudiantes universitarios y contraste por sexo de las variables antropométricas, prueba t de Student para las variables distribución normal y U de Mann-Whitney para la variable distribución no normal, significancia de $p<0.005$

$\mathrm{DE}=$ desviación estándar, $\mathrm{RIQ}=$ rango intercuartílico Fuente: Elaboración propia

En la figura 1 se muestra la clasificación del IMC por sexo, encontrando diferencias únicamente en la categoría de sobrepeso donde se observa que es mayor en los hombres $(\mathrm{p}=0.04)$.

\section{Figura 1. Clasificación de IMC por sexo de los} estudiantes universitarios

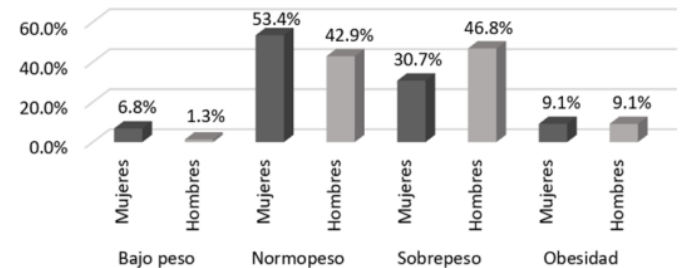

En la figura 2 se muestran los resultados por sexo de las preguntas del cuestionario KIDMED, en la que consumen 2 frutas diarias el $33.8 \%$ de los hombres y el $31.8 \%$ de las mujeres, y come verduras más de una vez al día el $39 \%$ y el $39,8 \%$, las respuestas a las que mayor porcentaje de estudiantes respondieron afirmativamente fueron el consumo de leguminosas más de una vez a la semana con un registro del $80.5 \%$ los hombres y $87.5 \%$ las mujeres, desayuna lácteos el $70.1 \%$ y el $71 \%$ respectivamente; de las preguntas con puntuación negativa, la pregunta si acude más de una vez a la semana a un restaurante de comida rápida la respuesta fue $45.5 \%$ y $45.6 \%$, no desayunan el $44.2 \%$ y el $33 \%$, desayuna bollería industrial $39 \%$ y $45 \%$ y consume varias veces al día dulces y bollería $27.3 \%$ y $40.9 \%$ respectivamente. No se observaron diferencias estadísticamente significativas en ninguna de las respuestas por sexo.

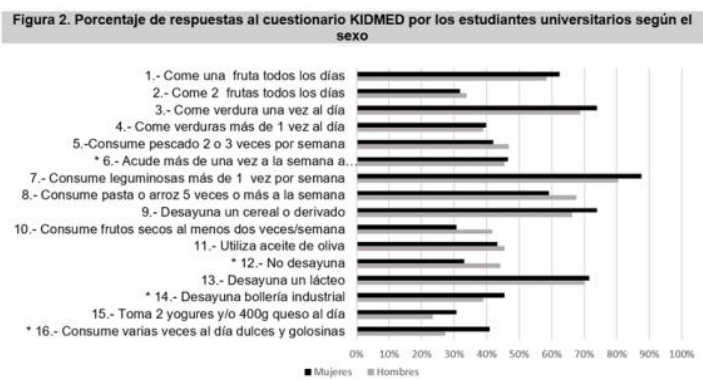

En la tabla 2 se observa que la adherencia a la dieta mediterránea de los estudiantes universitarios, "adherencia muy baja" el 33.9\%, con "necesidad de ajustes a los patrones mediterráneos" 48.5\% y "óptima" el $17.6 \%$, no se reflejaron diferencias estadísticamente significativas en la adherencia por sexo $(\mathrm{p}<.788)$.

Tabla 2. Adherencia de la dieta mediterránea por sexo de los estudiantes universitarios

\begin{tabular}{lcccccc}
\hline & \multicolumn{3}{c}{ Sexo } & \multicolumn{2}{c}{ Total } \\
Adherencia & No. & $\%$ & No. & $\%$ & No. & $\%$ \\
\hline Muy bajo & 29 & 33.0 & 27 & 35.1 & 56 & 33.9 \\
Necesidad de ajuste & 46 & 52.3 & 34 & 44.2 & 80 & 48.5 \\
Optima & 13 & 15 & 16 & 20.8 & 29 & 17.6 \\
Total & 88 & 100 & 77 & 100 & 165 & 100 \\
\hline
\end{tabular}

Fuente: Elaboración propia

Contraste por sexo a la adherencia con la prueba Chi cuadrado $(\mathrm{p}=0.014)$

\section{Discusión}

En la dieta mediterránea se recomienda el consumo cotidiano de agua, lácteos, verduras, frutas, leguminosas y carnes, por lo que sería conveniente que se incluya como parte de la enseñanza en la elección de alimentos.

La Encuesta Nacional de Salud y Nutrición 2018 menciona en el estado nutricional referido por el IMC, que el $36 \%$ de las mujeres de 20 años y mayores tienen sobrepeso y el $40 \%$ obesidad, mientras que los hombres el $42.4 \%$ y el $30.5 \%$ respectivamente (SSA/INSP, 2018), en este estudio 
el sobrepeso que se observa en los estudiantes evaluados es muy cercano al reportado a nivel nacional en adultos de 20 años o mayores, sin embargo, la obesidad es mucho menor en los estudiantes de esta investigación, pudiera ser por el rango de edad, que es menor. Landeros et al. (2018) reportaron en estudiantes universitarios de Guadalajara, Jalisco en México, una obesidad de 9\% que coincide con nuestro estudio.

En la búsqueda que realizamos conseguimos una publicación sobre la evaluación a la adherencia a la dieta mediterránea mediante el cuestionario KIDMED en estudiantes universitarios realizado en la Ciudad de México y aplicado por Gerardo et al. (2019), en el que reportan que los estudiantes evaluados no tienen adherencia a la dieta mediterránea y los estudiantes que realizan ejercicio físico consumen generalmente más verduras y tienen el hábito de desayunar.

En las frutas y verduras, la vitamina A o retinol proviene de los carotenos, principalmente del $\beta$ caroteno, ya que una sexta parte de este último se transforma en retinol, y frutas como la papaya, mango, durazno, melón y de verduras como las acelgas, las espinacas, la zanahoria y el brócoli, tienen un alto contenido de $\beta$-caroteno, por lo que es relativamente fácil completar los requerimientos de esta vitamina (Beltrán et al., 2012), también un consumo variado de frutas y verduras asegura una ingesta adecuada de nutrientes indispensables como la vitamina $\mathrm{C}$, la fibra y los compuestos fitoactivos (Pérez y Marivan, 2014), además, por su baja densidad calórica, ayudan a prevenir un aumento de peso corporal y disminuyen el riesgo de enfermedades no transmisibles (Calañas, 2005), es importante reconocer que una baja diversidad en las frutas y verduras consumidas, puede dificultar el mantenimiento de hábitos saludables a largo plazo (Mendonca et al., 2019); de las respuestas al cuestionario KIDMED por parte de los estudiantes universitarios estudiados, en las preguntas de "come 2 frutas todos los días" y "come verduras más de 1 vez al día”, un poco más de la tercera parte respondió que sí, lo que dificulta completar los requerimientos de estos nutrimentos en la mayoría de los estudiantes. Entre las preguntas de connotación negativa, está "si acude más de una vez por semana a restaurant de comida rápida", el $45.5 \%$ de los hombres y el $45.6 \%$ de la mujeres refirió que sí, de acuerdo con Sánchez $\&$ Aguilar (2015) este es un problema de salud ya que normalmente en estos lugares los alimentos de mayor disponibilidad son bollería elaborada con harina blanca, carne con alto contenido de grasa y azúcares, alimentos que consumiéndolos con cierta frecuencia pueden ser una de las causas de la obesidad, dislipidemias, hipertensión, diabetes y problemas cardiovasculares (Cruz et al., 2017). Los estudiantes universitarios tienen un ritmo de vida acelerado y no tienen el hábito para la elaboración de alimentos, es poco común que coman adecuadamente en casa, ya que pueden conseguir comida abundante y rápida a domicilio. Resultados similares obtuvo Bravo et al. (2019) donde el 35\% consumía comida rápida, principalmente papas fritas, debido a la disponibilidad y el sabor.

Las leguminosas, específicamente el frijol (Phaseolus vulgaris), que es de consumo popular en todo el país y además es el segundo cultivo que más se produce y se consume después del maíz (Zea mays L.), con una ingesta promedio anual per cápita de 10 kg (Ramírez et al., 2020), es rico en proteína, fibra, hierro y ácido fólico (Ulloa et al., 2011; Gómez \& Velázquez, 2019). Con relación a lo anterior, en este cuestionario los estudiantes respondieron que sí a la pregunta de "consume leguminosas más de una vez por semana", con el porcentaje más alto en todas las respuestas, un $80.5 \%$ los hombres y un $87.5 \%$ las mujeres.

En este estudio más de la mitad de los estudiantes contestaron que sí a la pregunta "consume frutos secos al menos dos veces por semana”, según Arias et al. (2019) estos alimentos son una fuente importante de antioxidantes como la vitamina $\mathrm{E}$ y el selenio, ricos en fitoquímicos y ácidos poliinsaturados que tienen un impacto importante y positivo en la neurogénesis, por eso existe una asociación entre el consumo de frutos secos y la función cognitiva, velocidad visual y memoria inmediata. El consumo de 30 gramos de frutos secos y aceite de oliva incluidos en una dieta saludable mejoran la función cognitiva (Martínez et al., 2013). Menos de la mitad de los estudiantes respondieron que sí a la pregunta "utiliza aceite de oliva", aun cuando se ha descubierto que tiene grandes beneficios su consumo, por ejemplo, Giacosa et al. (2016) sugieren que existe evidencia favorable sobre 
el riesgo de padecer cáncer en tracto aéreo y digestivo.

El desayuno es una comida importante ya que rompe un estado de ayuno del cuerpo, que se ha mantenido un tiempo considerable durante el periodo de sueño, en donde se reorganizan procesos metabólicos. Para considerarse que un desayuno es adecuado debe aportar por lo menos el $20 \%$ de los requerimientos energéticos diarios. De acuerdo con Uzhova et al. (2017), se ha visto que las personas que no desayunan tienen una dieta alejada al patrón de la dieta mediterránea, en el cuestionario KIDMED a la pregunta "no desayuna" el 38\% de los estudiantes que participaron en este estudio declararon no hacerlo. Saltarse el desayuno se ha visto relacionado con un riesgo mayor de padecer adiposidad, resistencia a la insulina y obesidad (López et al., 2018). No basta con desayunar todos los días, sino buscar un desayuno de calidad, que incluya que incluya los diferentes tipos de alimentos, y así aumente su calidad (Navarro et al., 2016).

Otra de las preguntas con mayor porcentaje de respuesta por los estudiantes es "desayuna un lácteo", el $70 \%$ de los hombres y el $71 \%$ de las mujeres respondieron afirmativamente, la región está entre las de mayor producción de lácteos en el país, participando con un 9.2\% (DIyEEyS, 2019), esta es una de las razones por la cual los lácteos forman parte de nuestra cultura alimentaria, aunque en la pregunta "consume 2 yogures y/o $400 \mathrm{~g}$ queso al día" solo el $23.4 \%$ de los hombres y el $30.7 \%$ de las mujeres respondió que sí, quizá por la cantidad específica de la pregunta. De acuerdo con Navarro et al. (2016) es conveniente ingerir lácteos desnatados o reducidos en azúcar a pesar de ser un alimento alto en proteína, calcio y vitaminas, ya que hay una inclinación a padecer obesidad si se ingieren lácteos con grandes cantidades de azúcar.

A la pregunta "consume varias veces al día dulces y golosinas" aproximadamente la tercera parte de los estudiantes reportaron que sí, estos resultados se asemejan al estudio de Navarro (2017), donde describe que estos alimentos proporcionan una sensación de placer y satisfacción, aportan azúcares simples y aditivos con grandes cantidades de sacarosa o jarabe de fructosa, también Castañeda et al. (2016) refieren que se consideran alimentos industrializados desbalanceados con alto contenido de carbohidratos, grasas, sales y pobres en vitaminas. La dieta mediterránea es uno de los modelos que más ha demostrado sus beneficios en la salud del ser humano, se considera como un tratamiento preventivo y primario en diversas enfermedades, como las cardiovasculares, alérgicas y psiquiátricas, además mejorar la calidad de vida (Serra \& Ortiz, 2018), también se ha relacionado con menor riesgo de deterioro cognitivo, de enfermedad de Alzheimer y otras formas de demencia, mejorando la función cognitiva en adultos mayores (Pooja \& Dahl, 2018). En este estudio solo el $17.6 \%$ de los jóvenes universitarios tuvieron una adherencia óptima a la dieta mediterránea, obteniendo resultados similares a los de García et al. (2015), donde se mostró una baja adherencia a la dieta mediterránea en niños y jóvenes del mediterráneo, con tendencia al abandono, previendo que esta situación puede traer eventos adversos a la salud dada la efectividad del patrón dietético de la dieta mediterránea.

Para estudios posteriores sería conveniente ampliar la muestra a los estudiantes universitarios de todos los semestres, para que la muestra sea representativa estadísticamente, a su vez se recomienda usar herramientas antropométricas de evaluación de la composición corporal que diferencien entre masa libre de grasa y masa grasa, ya que al tratarse de estudiantes de la cultura física, estos pudieran tener una musculatura más desarrollada, causada por hábitos de cuidado y mantenimiento de la salud como la práctica de ejercicio o deporte.

\section{Conclusiones}

La mayoría de los estudiantes universitarios necesitan de ajustes en su dieta a los patrones mediterráneos, por lo que se debe hacer mayor promoción a los beneficios de corto y largo plazo que ofrece la dieta mediterránea respecto a la dieta occidental, parte de estos trabajos es la motivación por constituir un programa de formación integral hacia los estudiantes que profesionalmente se dedicarán a la actividad física, para que lleven un control de su alimentación, lo cual les brindará beneficios personales y familiares.

\section{Bibliografía}

Arias, L., López, E., Struijk, E. A., Rodríguez, F. \& Lana, A. (2019). Consumo de frutos secos y función cognitiva: una revisión sistemática. 
Nutrición Hospitalaria, 36(5), 1179-1188. doi:10.20960/nh.02566 Recuperado de http://scielo.isciii.es/pdf/nh/v36n5/1699-5198nh-36-05-01179.pdf

Arriaga, J. C. P., \& Cruz, S. E. (2019). La obesidad desde la perspectiva de la selección de alimentos. Revista Salud Pública y Nutrición, 18(1), 25-32. doi:10.29105/respyn18.1-4. Recuperado de http://respyn.uanl.mx/index.php/respyn/article/vi ew/417/344

Arroyo, M., Rocandio, A. M., Ansotegui, L., Pascual, E., Salces, I., \& Rebato, E. (2006). Calidad de la dieta, sobrepeso y obesidad en estudiantes universitarios. Nutrición hospitalaria, 21(6), 673-679. Recuperado de http://scielo.isciii.es/pdf/nh/v21n6/original6.pdf

Avalos, M. B. B., Salazar, J. L. L., Matveev, L. A. V., \& Miranda, M. F. (2020). Comercialización, consumo y valor nutricional de la comida rápida, en los estudiantes universitarios, caso: carrera de agroindustria de la facultad de ciencias pecuarias/Marketing, consumption and nutritional value of rapid food, in university students. KnE Engineering, 94-106. doi:10.18502/keg.v5i2.6225. Recuperado de https://knepublishing.com/index.php/KnEEngineering/article/view/6225/11597

Babio, N., Bullo, M., Basora, J., Martínez, M. A., Fernández, J., Márquez, F., Molina, C., \& Salas, J. (2009). Adherence to the Mediterranean diet and risk of metabolic syndrome and its components. Nutrition, Metabolism and Cardiovascular Diseases, 19(8), 563-570. doi:10.1016/j.numecd.2008.10.007. Recuperado de

https://www.sciencedirect.com/science/article/ab s/pii/S0939475308002226

Beltrán, B., Estévez, R., Cuadrado, C., Jiménez, S., \& Olmedilla, A. B. (2012). Base de datos de carotenoides para valoración de la ingesta dietética de carotenos, xantofilas y de vitamina A; utilización en un estudio comparativo del estado nutricional en vitamina $\mathrm{A}$ de adultos jóvenes. Nutrición Hospitalaria, 27(4), 1334-1343. doi:10.3305/nh.2012.27.4.5886. Recuperado de http://hdl.handle.net/10261/54030

Calañas, A. J. (2005). Alimentación saludable basada en la evidencia. Endocrinología y Nutrición, 52, 8-24. doi:10.1016/S1575-0922(05)74649-0. Recuperado de https://www.sciencedirect.com/science/article/ab $\underline{\mathrm{s} / \mathrm{pii} / \mathrm{S} 1575092205746490}$

Castañeda, E., Ortiz, H., Robles, G., \& Molina, N. (2016). Consumo de alimentos chatarra y estado nutricio en escolares de la Cuidad de México. Revista Mexicana de Pediatría, 83(1), 15-19. Recuperado https://www.medigraphic.com/pdfs/pediat/sp2016/sp161d.pdf

Cruz, M., Sánchez, N. A., Dávila, G., \& Jiménez, C. (2017). Aspectos evolutivos de la alimentación básica de la población mexicana y su efecto en la obesidad. En M.E. Ramírez Ortiz (Ed.). Alimentos Funcionales de Hoy. Barcelona, España: OmniaScience Monographs. 247-284.

Díaz, I., Gascón, E., Lázaro, S., \& Maximiano, C. (2007). Guía de la alimentación mediterránea. Ed. Empresa Pública Desarrollo Agrario y Pesquero. Consejería de Agricultura y Pesca. Junta de Andalucía.

Dirección de Investigación y Evaluación Económica y Sectorial. (2019). Panorama Agroalimentario. Leche y Lácteos. FIRA. Recuperado de https://www.inforural.com.mx/wpcontent/uploads/2019/06/PanoramaAgroalimentario-Leche-y-la769cteos-2019.pdf

Dussaillant, C., Echeverría, G., Urquiaga, I., Velasco, N., \& Rigotti, A. (2016). Evidencia actual sobre los beneficios de la dieta mediterránea en salud. Revista médica de Chile, 144(8), 1044-1052. doi: 10.4067/S003498872016000800012. Recuperado de https://scielo.conicyt.cl/pdf/rmc/v144n8/art12.pd $\underline{\mathrm{f}}$

Escaffi, M. J., Miranda, M., Alonso, R., \& Cuevas, A. (2016). Dieta mediterránea y vitamina D como potenciales factores preventivos del deterioro cognitivo. Revista Médica Clínica Las 
Condes, 27(3),

392-400. doi:10.1016/j.rmclc.2016.06.012. Recuperado de https://www.sciencedirect.com/science/article/pi i/S0716864016300396

Ferrís, J., Berbel, O., García, J., Ortega, J. A., \& López, J. A. (2012). Factores dietéticos asociados al cáncer de próstata. Beneficios de la dieta mediterránea. Actas Urológicas Españolas, $36(4)$, 239-245. doi:10.1016/j.acuro.2011.08.002. Recuperado de https://www.sciencedirect.com/science/article/ab s/pii/S0210480611003202

García, S., Fernández, N., Rodríguez, C., Nissensohn, M., Román, B., \& Serra, L. (2015). KIDMED test; prevalence of low adherence to the Mediterranean Diet in children and young; a systematic review. Rev. Nutrición Hospitalaria, $32(6)$, 2390-2399. doi:10.3305/nh.2015.32.6.9828. Recuperado de https://www.redalyc.org/pdf/3092/30924332100 $\underline{7 . p d f}$

Gerardo, F. R., Martínez, E. A., Rosales, L. F., Soto, D. M., Tadeo, C., Sámano, M. R., \& Méndez, S. (2019). Calidad Alimentaria y Estilo de Vida En Estudiantes Universitarios de $\mathrm{La}$ Ciudad de México. REDNUTRICIÓN, 10(3), 668-674. Recuperado https://www.researchgate.net/profile/LauraRosalesArreola/publication/345392472_Calidad_alimen taria_y_estilo_de vida_en estudiantes_universit arios de la_Ciudad_de_Mexico/links/5fa59f979 2851cc2869cca67/Calidad-alimentaria-y-estilode-vida-en-estudiantes-universitarios-de-laCiudad-de-Mexico.pdf

Giacosa, A., Barale, R., Bavaresco, L., Gatenby, P., Gerbi, V., Janssens, J., ... \& Rondanelli, M. (2013). Cancer prevention in Europe: the Mediterranean diet as a protective choice. European journal of cancer prevention, 22(1), 90-95. doi: 10.1097/CEJ.0b013e328354d2d7 From https://journals.lww.com/eurjcancerprev/Fulltext /2013/01000/Cancer_prevention_in_Europe he_Mediterranean.13.aspx
Gómez, J., \& Velázquez, E. B. (2019). Salud y Cultura Alimentaria en México. Revista Digital Universitaria, 20(1), 1-11. doi:10.22201/codeic.16076079e.2019.v20n1.a6. Recuperado de http://www.revista.unam.mx/wpcontent/uploads/v20_n1_a6 Salud-y-culturaalimentaria-en-Mexico.pdf

Gómez, Z., Landeros, P., Romero, E., \& Troyo, R. (2016). Estilos de vida y riesgos para la salud en una población universitaria. Revista Salud Publica y Nutrición, 15(2) Recuperado de http://respyn.uanl.mx/index.php/respyn/article/vi $\underline{\mathrm{ew} / 11 / 11}$

Landeros, R. P., Gómez, C. Z., Rimoldi, R. M. J., Parada, B. G., \& Núñez, A. (2018). Índice de masa corporal y rendimiento académico en estudiantes universitarios. Revista Salud Pública y Nutrición, 17(4), 34-40. Recuperado de https://www.medigraphic.com/pdfs/revsalpubnut /spn-2018/spn184d.pdf

López, A. M., Cuadrado, E., Peral, Á., Aparicio, A., Ortega, R. M., López, A. M., \& Ortega, R. M. (2018). Importancia del desayuno en la mejora nutricional y sanitaria de la población. Nutrición Hospitalaria, 35. doi:10.20960/nh.2278, Recuperado de https://pdfs.semanticscholar.org/3104/0f96d81b $\underline{\text { 8cb0465bb359158ebcacf80f76bf.pdf }}$

Martínez, E. H., Clavero, P., Toledo, E., Estruch, R., Salas, J., San-Julian, B., Sánchezx-Tainta, A., Ros, E., Vall, C., \& Martínez, M. A. (2013). Mediterranean diet improves cognition: the PREDIMED-NAVARRA randomised trial. Neurosurgery y Physchiatry, 84(12), 1318-1325. doi: $\quad 10.1136 /$ jnnp-2012-304792 from https://jnnp.bmj.com/content/84/12/1318.short

Mendonça, R. D. D., Souza, L. M., Pinheiro, P., Campos, S. F., Carvalho, M., \& Souza, A. C. (2019). Monotony in the consumption of fruits and vegetables and food enviroment characteristics. Revista Saúde Pública, 53, 63. doi: $\quad 10.11606 / S 1518-8787.2019053000705$. from https://www.scielosp.org/pdf/rsp/2019.v53/63/e n/ 
Navarro, I., Periago, M. J., \& García, F. (2017). Estudio de nuevas fórmulas de golosinas de bajo índice glucémico. Revista Española Nutrición Comunitaria, 23(4). Recuperado de http://renc.es/imagenes/auxiliar/files/RENC_201 7_4 6. I Navarro.pdf

Navarro, I., Ros, G., Martínez, B., Rodríguez, A., \& Periago M. (2016). Adherencia a la dieta mediterránea y su relación con la calidad del desayuno en estudiantes de la Universidad de Murcia. Nutrición Hospitalaria, 33(4), 901-908. doi:10.20960/nh.390. Recuperado de http://scielo.isciii.es/scielo.php?script=sci_arttex $\underline{\mathrm{t} \& \mathrm{pid}=\mathrm{S} 0212-16112016000400021}$

Organización de las Naciones Unidas para la Educación la Ciencia y la Cultura. (2013) La dieta mediterránea $\left(\mathrm{n}^{\circ} 00884\right)$. Recuperado de https://ich.unesco.org/es/RL/la-dietamediterranea-00884

Peñalvo, J. L., Oliva, B., Sotos, M., Uzhova, I., Moreno, B., León, M., \& Ordovás, J. M. (2015). La mayor adherencia a un patrón de dieta mediterránea se asocia a una mejora del perfil lipídico plasmático: la cohorte del Aragon Health Workers Study. Revista Española de Cardiología, $68(4)$, 290-297. doi:10.1016/j.recesp.2014.09.018. Recuperado de

https://www.sciencedirect.com/science/article/ab s/pii/S0300893214006125

Pérez Lizaur, A. B., L Marivan Laborde. (2014). Sistema Mexicano de Equivalentes. Fomento de Nutrición y Salud A.C. $4^{\circ}$ ed. México.

Ponce, P. L., Ruiz, J., Magaña, A., Arizona, B.., \& Mayagoitia, J. (2011). Obesidad y factores de riesgo en estudiantes del área de la salud de la universidad autónoma de baja california, Mexicali. Revista Salud Publica y Nutrición 12(4). Recuperado de http://respyn.uanl.mx/index.php/respyn/article/vi $\underline{\mathrm{ew} / 300}$

Pooja, T., Dahl, W. (2018). La Dieta y la Salud del Cerebro. Food Science and Human Nutrition,
18(2), 1-3. doi:10.32473/edis-fs307-2018. Recuperado de https://journals.flvc.org/edis/article/view/105721 1118780

Ramírez, R., Palacios, N., Nutti, M., \& Pérez, S. (2020). Estados potenciales en México para la producción y consumo de frijol biofortificado con hierro y zinc. Revista Fitotecnia Mexicana, 43(1), 11-23. doi:10.35196/rfm.2020.1.11. Recuperado de https://revfitotecnia.mx/index.php/RFM/article/v iew/309/283

Sánchez, V., \& Aguilar, A. (2015). Hábitos alimentarios y conductas relacionadas con la salud en una población universitaria. Nutrición Hospitalaria, 31(1), 449-457. doi: 10.3305/nh.2015.31.1.7412. Recuperado de http://scielo.isciii.es/pdf/nh/v31n1/51originalval oracionnutricional03.pdf

Secretaría de Salud, Instituto Nacional de Salud Pública (2018). Encuesta Nacional de Salud y Nutrición (ENSANUT 2018). Presentación de resultados. Recuperado de https://ensanut.insp.mx/encuestas/ensanut2018/d octos/informes/ensanut_2018_presentacion_resu ltados.pdf

Serra, L., \& Ortiz, A. (2018). La dieta mediterránea como ejemplo de una alimentación y nutrición sostenibles: enfoque multidisciplinar. Nutrición Hospitalaria, 35(4), 96-101. doi: 10.20960/nh.2133. Recuperado de https://europepmc.org/article/med/30070130

Serra, L., Ribas, L., Ngo, J., Ortega, R. M., García, A., Pérez, C., \& Aranceta J. (2004). Food, youth and the Mediterranean Diet in Spain. Development of KIDMED, Mediterranean Diet Quality Index in children and adolescents. Public Health Nutrition, 7(7), 931-935. doi:10.1079/PHN2004556 from https://www.cambridge.org/core/journals/publichealth-nutrition/article/food-youth-\%20and-themediterranean-diet-in-spain-development-ofkidmedmediterraneandietqualityindexinchildrenandadol escents/BDC8AE7999DB6E4F234A9098F8927 C84 
Thomas, D. T., Erdman, K. A., \& Burke, L. M. (2016). American College of Sports Medicine Joint Position Statement. Nutrition and Athletic Performance. Medicine and science in sports and exercise, 48(3). 543-568. doi: 10.1249/MSS.0000000000000852. from https://www.migliaccio.it/wpcontent/uploads/2018/07/Nutrition_and_Athletic Performance.25.pdf

Ulloa, J. A., Ulloa, P. R., Ramírez, J.C. \& Ulloa, B. E. (2011). El frijol (Phaseolus vulgaris): su importancia nutricional y como fuente de fitoquimicos. CONACYT, 3(8), 5-9. Recuperado de http://192.100.162.123:8080/bitstream/1234567 89/582/1/E1\%20frijol\%20\%28Phaseolus\%20vul garis $\% 29 \% 2 \mathrm{c} \% 20 \mathrm{su} \% 20 \mathrm{importancia} \% 20$ nutrici onal.pdf

Uzhova I, Fuster, V., Fernández, A., Ordovás, J. M., Sanz, J., Fernández,,L., López-Melgar, B., Mendiguren, J. M., Bueno, H., \& Peñalvo, J. L. (2017). The Importance of Breakfast in Atherosclerosis Disease. Insigths From the Pesa Study. Journal of the American College of Cardiology, 70(15), 1833-1842. doi: 10.1016/j.jacc.2017.08.027. from https://www.onlinejacc.org/content/accj/70/15/1 833.full.pdf

Van, N. A., Larson, N., \& Laska, M. N. (2012). Emerging adulthood: a critical age for preventing excess weight gain? Adolescent medicine: state of the art reviews, 23(3), 571-588. From https://experts.umn.edu/en/publications/emergin g-adulthood-a-critical-age-for-preventingexcess-weight-ga

World Health Organization (1995) Physical status: the use and interpretation of anthropometry: report of a WHO Expert Committee. WHO Technical Report Series, 854. From https://apps.who.int/iris/bitstream/handle/10665/ 37003/WHO_TRS_854.pdf 